\title{
On a conjecture of Erdős, Pólya and Turán on consecutive gaps between primes
}

\author{
János Pintz*
}

\section{Introduction}

One of the favourite problems of Erdős (and Turán) was to investigate local problems in the distribution of primes, in particular to examine gaps or blocks of successive gaps between consecutive primes.

Let $\mathcal{P}:=\left\{p_{n}\right\}_{1}^{\infty}$ be the sequence of all primes and

$$
d_{n}=p_{n}-p_{n-1} \quad(n=2,3, \ldots)
$$

be the sequence of gaps between consecutive primes.

In 1948 Erdős and Turán [4] showed that

$$
d_{n+1}-d_{n}
$$

changes sign infinitely often. Soon after this Erdős [2] showed the stronger relation

$$
\liminf _{n \rightarrow \infty} \frac{d_{n+1}}{d_{n}}<1<\limsup _{n \rightarrow \infty} \frac{d_{n+1}}{d_{n}} .
$$

In the same work [4], that is, already 67 years ago, Erdős and Turán asked for a necessary and sufficient condition that

$$
\sum_{i=1}^{k} a_{i} p_{n+i}
$$

should have infinitely many sign changes as $n \rightarrow \infty$, where $a_{1}, \ldots, a_{k}$ are given real numbers. They observed that

$$
\sum_{i=1}^{k} a_{i}=0
$$

*Supported by ERC-AdG. 321104 and OTKA NK104183. 
is clearly necessary, and Pólya observed that if (1.4) has infinitely many sign changes, then the $k$ numbers

$$
\alpha_{j}=\sum_{i=1}^{j} a_{n}
$$

cannot all have the same sign. As described in [4] and [3], Erdös, Pólya and Turán then conjectured that the above condition on $\alpha_{j}$ is a necessary and sufficient condition for the infinitely many sign changes of (1.4). As Erdös writes on p. 12 of [3]: "We are very far from being able to prove this, in fact I cannot even prove that $d_{n}>d_{n+1}+d_{n+2}$ has infinitely many solutions. I proved the following much easier theorem: Assume that

$$
\sum_{i=1}^{k-1} \alpha_{i}=0 \quad \text { and } \quad \alpha_{k-1} \neq 0 .
$$

Then (1.4) changes sign infinitely often."

In my recent work [7] I showed several partial results in this direction (see Theorems 17-19) but I was far from being able to show the original conjecture of Erdős, Pólya and Turán. In the present work I will show the original conjecture based on the recent groundbreaking ideas of J. Maynard [6] and T. Tao [9] on bounded gaps between primes.

\section{Some remarks and a stronger form of the Erdős- Pólya-Turán conjecture}

Since the necessity of (1.5) is trivial we can further on always suppose (1.5). So we can rewrite (1.4) as

$$
\begin{aligned}
T & =\sum_{i=1}^{k} a_{i} p_{n+i}=\sum_{i=1}^{k} a_{i}\left(p_{n}+\sum_{\nu=1}^{i} d_{n+\nu}\right)=\sum_{i=1}^{k} a_{i}\left(\sum_{\nu=1}^{i} d_{n+\nu}\right) \\
& =\sum_{j=1}^{k} d_{n+j} \sum_{i=j}^{k} a_{i}=-\sum_{j=1}^{k} d_{n+j} \alpha_{j-1}=-\sum_{j=2}^{k} d_{n+j} \alpha_{j-1}
\end{aligned}
$$

if we define $\alpha_{0}=0$.

Thus the original conjecture is equivalent to the following one (if we let $\ell:=k-1)$. 
Conjecture (Erdős-Pólya-Turán). The expression

$$
\sum_{i=1}^{\ell} \alpha_{i} d_{n+i}
$$

changes sign infinitely often as $n$ runs through all integers if and only if the non-zero elements among $\alpha_{1}, \alpha_{2}, \ldots, \alpha_{\ell}$ do not all have the same sign.

Theorem 1. The above conjecture is true.

The above theorem clearly follows from (but as it is easy to see, is in fact equivalent to) the following one.

Theorem 2. We have for every fixed natural number $\ell$

$$
\limsup _{m \rightarrow \infty} \frac{d_{m}}{\max \left(d_{m-\ell}, \ldots, d_{m-1}, d_{m+1}, \ldots, d_{m+\ell}\right)}=\infty .
$$

We will prove this in the stronger form expressed by

Theorem 3. For every natural number $\ell$ there exists an explicitly calculable constant $c(\ell)>0$ such that

$$
\limsup _{m \rightarrow \infty} \frac{d_{m}}{(\log m)^{c(\ell)} \max \left(d_{m-\ell}, \ldots, d_{m-1}, d_{m+1}, \ldots, d_{m+\ell}\right)}>0 .
$$

Remark. It follows from the proof that one can take

$$
c(\ell)=C_{1} e^{-C_{2} \ell}
$$

with positive absolute constants $C_{1}, C_{2}>0$.

\section{Proof of Theorem 3}

The proof will be based on the first assertion (see (i)) of a very nice result of W. D. Banks, T. Freiberg and J. Maynard [1] which appears as Theorem 4.3 in their work, which we quote now restricted on (i) and with a slight change as

Theorem A (Banks-Freiberg-Maynard). Let $m, k$ and $\epsilon=\epsilon(k)$ be fixed. If $k$ is a sufficiently large multiple of $16 m+1$ and $\epsilon$ is sufficiently small, there is 
some $N(m, k, \epsilon)$ such that the following holds for all $N \geqslant N(m, k, \epsilon)$. With $Z_{N^{4 \epsilon}}$ given by $(4.8)$ of [1], let

$$
w=\epsilon \log N \text { and } W=\prod_{\substack{p \leqslant w \\ p \nmid Z_{N^{4 \epsilon}}}} p .
$$

Let $\mathcal{H}=\left\{h_{1}, \ldots, h_{k}\right\}$ be an admissible $k$-tuple such that

$$
0 \leqslant h_{1}, \ldots, h_{k} \leqslant N
$$

and

$$
p \mid \prod_{1 \leqslant i<j \leqslant k}\left(h_{j}-h_{i}\right) \Longrightarrow p \leqslant w .
$$

Let

$$
\mathcal{H}=\mathcal{H}_{1} \cup \cdots \cup \mathcal{H}_{16 m+1}
$$

be a partition of $\mathcal{H}$ into $16 m+1$ sets of equal size. Finally, let $b$ be an integer such that

$$
\left(\prod_{i=1}^{k}\left(b+h_{i}\right), W\right)=1 .
$$

There is some $n_{1} \in(N, 2 N]$ with $n_{1} \equiv b \bmod W$, and some set of $m+1$ distinct indices $\left\{i_{1}, \ldots, i_{m+1}\right\} \subseteq\{1, \ldots, 16 m+1\}$, such that

$$
\left|\mathcal{H}_{i}\left(n_{1}\right) \cap \mathbb{P}\right|=1 \text { for all } i \in\left\{i_{1}, \ldots, i_{m+1}\right\} \text {. }
$$

Remark 1. The definition of $Z_{N^{4 \epsilon}}$ is given earlier in the work [1] but its value does not play a significant role in the application of the result (it is the greatest prime factor of a possible exceptional modulus if such a modulus exists and it is equal to 1 if no such modulus exists).

Remark 2. According to the calculation of the present author $8 m+1$ in (4.18) of [1] has to be replaced by $16 m+1$.

The proof uses the Maynard-Tao method [6, [9] and other important ideas as a modified Erdős-Rankin type construction (see Section 5 of [1]), a modified Bombieri-Vinogradov theorem, somewhat similar to Theorem 6 of [5], and an important observation of the Polymath project [8] according to which one can estimate from above how often we have more than one prime in the translation of a subset $\mathcal{H}^{\prime}$ of an admissible $k$-tuple $\mathcal{H}$ (in the weighted sense). 
We note that the variable $k$ in Theorem $\mathrm{A}$ has nothing to do with the one appearing in Section 1 and (2.1) of our work which satisfied $k=\ell+1$. The present $k$ will be here a large multiple of $16 m+1$ and $m$ will satisfy $m \asymp \ell$ here. In fact we will define now

$$
L:=\ell+2, \quad m:=62 L-33=62 \ell+91 .
$$

The Maynard-Tao method needs to choose in the proof of Theorem A

$$
k=\exp \left(C_{3} m\right)=\exp \left(C_{4} \ell\right)
$$

(by the relation $\delta \varrho \log k=2 m$, appearing in the first line on p. 17 of [1]). This will imply the appearance of $(\log n)^{c(\ell)}$ in (2.4) of us with $c(\ell)$ defined as in (2.5), that is $C_{1} \exp \left(-C_{2} \ell\right)$.

In order to show Theorem 3 we will choose with a sufficiently large $k$ an admissible $k$-tuple of $\mathcal{H}$ with

$$
2(16 m+1) \mid k
$$

for every given sufficiently large $N$. We further let

$$
J:=32 L-17
$$

which implies

$$
16 m+1=992 L-527=31 J .
$$

We will partition our admissible $k$-tuple $\mathcal{H}$ into $16 m+1=31 J$ subsets of equal size $k /(31 J)$.

We will use the additional information of [1] (see Sections 5 and 6 of it) that by the Erdős-Rankin procedure one can find for any sufficiently large $N$ an admissible $k$-tuple $\mathcal{H}$ and a number $n \in[N, 2 N]$ which we fix in the following, such that with a

$$
z>\log N \sqrt{\log _{2} N}
$$

all numbers of the form

$$
n+\nu, \quad 1<\nu \leqslant z, \quad \nu \notin \mathcal{H}
$$

should be composite. Hence all possible primes in $(n+1, n+z]$ should be of type $n+h_{i}, h_{i} \in \mathcal{H}$. We have here a lot of freedom in choosing $\mathcal{H}$. First its 
elements can be as large as $\log N$ and the conditions of Theorem A allow us to choose its elements as

$$
h_{i}=\sum_{j=1}^{i} b_{j} \quad \text { and } \quad b_{i}=(1+o(1)) \beta_{j}(\log N)^{c} j
$$

for any choice of $\beta_{1}, \ldots, \beta_{k}$ and $c_{1}, \ldots, c_{k} \in(0,1]$ (see Sections 5-6 of [1]). We will choose $\beta_{i}=1(i=1,2, \ldots, k)$ and with

$$
K=\frac{k}{62 J}, \quad i=(\nu J+\mu) K+\lambda, \quad 0 \leqslant \nu \leqslant 30, \quad 0 \leqslant \mu \leqslant J-1, \quad 1 \leqslant \lambda \leqslant K
$$

we will choose

$$
c_{i}=f(\nu, \mu, \lambda):=\frac{(30-\nu) J+\mu}{62 J}+\frac{\lambda}{k} \in\left[\frac{1}{k}, \frac{1}{2}\right] .
$$

This means that

$$
h_{j}-h_{i}=\sum_{t=i+1}^{j} b_{t} \sim \max _{i<t \leqslant j} b_{t} \quad \text { for } i<j .
$$

From (3.16) we further see that $c_{i}=f(\nu, \mu, \lambda)$, and consequently $b_{i}$ will be monotonically increasing in both $\mu$ and $\lambda$ for each fixed value of $\nu$ when $\mu \in[0, J-1], \lambda \in[1, K]$. More exactly, for every fixed $\nu$ we have

$$
f(\nu, \mu, \lambda)-f\left(\nu, \mu^{\prime}, \lambda^{\prime}\right) \geqslant \frac{1}{k} \text { if } \mu>\mu^{\prime} \text { or } \mu=\mu^{\prime}, \lambda>\lambda^{\prime} .
$$

On the other hand this construction shows that

$$
f\left(\nu_{2}, \mu_{2}, \lambda_{2}\right)-f\left(\nu_{1}, \mu_{1}, \lambda_{1}\right)=\frac{\nu_{1}-\nu_{2}}{62}+\frac{\mu_{2}-\mu_{1}}{62 J}+\frac{\lambda_{2}-\lambda_{1}}{k} \leqslant-\frac{1}{k}
$$

if $\nu_{2}>\nu_{1}$ for every quadruple $\left(\mu_{1}, \mu_{2}, \lambda_{1}, \lambda_{2}\right)$ if $\mu_{i} \in[0, J-1], \lambda_{i} \in[1, K]$ which means that $f(\nu, \mu, \lambda)$ is monotonically decreasing in $\nu$ independently of the values of $\mu$ and $\lambda$.

Let us define now the partition of $\mathcal{H}$ into $31 J=16 m+1$ subsets $\mathcal{H}_{\nu, \mu}$ $(0 \leqslant \nu \leqslant 30,0 \leqslant \mu \leqslant J-1)$ as

$$
\mathcal{H}_{\nu, \mu}:=\left\{h_{i}\right\}_{i \in I_{\nu, \mu}} I_{\nu, \mu}=\{i=(\nu J+\mu) K+\lambda, 1 \leqslant \lambda \leqslant K\}
$$

and let us organize these subsets into 31 columns according to the value of the index $\nu$ for $\nu=0,1, \ldots, 30$. 
The observations (3.17)-(3.19) show that the values of $b_{i}$ are increasing (by a factor $\left.\geqslant(1+o(1))(\log N)^{1 / k}\right)$ within each column. Further we see that if $b_{i}$ is in another column than $b_{j}$ with an index

$$
\nu(i)<\nu(j)
$$

then necessarily

$$
\frac{b_{i}}{b_{j}} \geqslant(\log N)^{1 / k}(1+o(1)) .
$$

This means by (3.17) that if $n+h_{i}$ and $n+h_{j}(i<j)$ are consecutive primes, then their difference is asymptotically equal to the dominant $b_{t}$ with $i<t \leqslant j$ and the ratio between two consecutive primegaps will be

$$
\geqslant(\log N)^{1 / k}(1+o(1)) \text { or } \leqslant(1+o(1))(\log N)^{-1 / k} .
$$

Theorem 2 will be shown if we can reach in one of the columns with index $\nu=0,1, \ldots, 29$ (that is, $\nu \neq 30$ ) at least $L$ primes of the form $n+h_{i}$ in such a way that we should have still in total at least $L$ primes of type $n+h_{i}$ in all remaining columns with an index larger than $\nu$.

In this case we can choose the largest index $i$ within that column (that is, with $\nu(i)=\nu$ ) as our $h_{i}$ for which $n+h_{i} \in \mathcal{P}$, and we let

$$
p_{m}:=n+h_{i} .
$$

This will imply that we have additionally $p_{m-1}, \ldots, p_{m-L+1}=p_{m-\ell-1}$ in the same column and the differences $d_{m}, d_{m-1}, \ldots, d_{m-\ell}$ satisfy

$$
\frac{d_{m-i}}{d_{m-j}} \gg(\log N)^{(j-i) / k} \text { for } 0 \leqslant i<j \leqslant \ell
$$

in accordance with (2.4).

Further, in view of (3.21)-(3.22), as all the later primes of type $p_{m+t}$ with $t \geqslant 1$ are of the form $n+h_{j}$ with $\nu(j)>\nu(i)$ we will have for the increments the relation (3.22) and this will yield

$$
\frac{d_{m}}{d_{m+t}} \gg(\log N)^{1 / k} \text { for } 0<t, p_{m+t} \leqslant n+z .
$$

So, let us suppose now that the first column having at least $L$ primes of the form $n+h_{i}$ has index $y$, where $0 \leqslant y \leqslant 30$. If such an index, that is, such a column does not exist, then we have in total at most

$$
31 L<62 L-32=m+1
$$


primes among $n+h_{i}$ in contradiction with (3.6) in Theorem A. So we have such a column with index $y \in[0,30]$. This column contains at most $J$ subsets of type $\mathcal{H}_{i_{t}}$ described in (3.6). If we have no further column at all (i.e. $y=30$ ) or the number of primes in later columns is in total at most $L-1$, then we have in total at most $30(L-1)$ primes in all other columns. This means that the total number of subsets $\mathcal{H}_{i_{t}}$ with exactly one prime of the form $n+h_{j}$ in it $\left(h_{j} \in \mathcal{H}_{i_{t}}\right)$ is at most (cf. (3.6), (3.7) and (3.10) )

$$
30(L-1)+J=62 L-47<62 L-32=m+1
$$

in contradiction with (3.6) in Theorem A. Together with the earlier observations (3.21) - (3.26) this shows Theorem 3 and consequently Theorem 2 and Theorem 1, the conjecture of Erdős, Pólya and Turán.

\section{References}

[1] W. D. Banks, T. Freiberg, J. Maynard, On limit points of the sequence of normalized prime gaps, arXiv:1404.5094 2

[2] P. Erdős, On the difference of consecutive primes, Bull. Amer. Math. Soc. 54 (1948), 885-889.

[3] P. Erdős, Problems and results in number theory. In: Recent progress in analytic number theory, Vol. 1 (Durham, 1979), pp. 1-13, Academic Press, London-New York, 1981.

[4] P. Erdős, P. Turán, On some new questions on the distribution of prrime numbers, Bull. Amer. Math. Soc. 54 (1948), 371-378.

[5] D. A. Goldston, J. Pintz, C. Y. Yıldırım, Primes in tuples II, Acta Math. 204 (2010), 1-47.

[6] J. Maynard, Small gaps between primes, Ann. of Math. (2) 181 (2015), no. 1, 383-413.

[7] J. Pintz, Paul Erdős and the difference of primes. In: Erdös centennial, pp. 485-513, Bolyai Soc. Math. Stud., 25, János Bolyai Math. Soc., Budapest, 2013.

[8] D. H. J. Polymath, Variants of the Selberg sieve, and bounded intervals containing many primes, arXiv:1407.4897v 4

[9] T. Tao, unpublished manuscript. 
János Pintz

Rényi Mathematical Institute

of the Hungarian Academy of Sciences

Budapest, Reáltanoda u. 13-15

H-1053 Hungary

e-mail:pintz.janos@renyi.mta.hu 